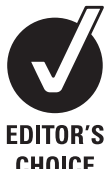

CHOICE

\title{
Medication management in people with Parkinson's disease during surgical admissions
}

\author{
C P Derry, K J Shah, L Caie, C E Counsell
}

Aberdeen Royal Infirmary, Aberdeen, UK

\section{Correspondence to} Dr C Derry, Aberdeen Royal Infirmary, Foresterhill Rd, Aberdeen AB25 2ZN, UK; cderry@nhs.net

Received 18 February 2009 Accepted 29 November 2009

\begin{abstract}
Background Patients with Parkinson's disease (PD) may experience problems in hospital, with their medication being withheld or inappropriate medication being prescribed. Since surgical admissions present particular risks, the authors examined the management of patients with PD on surgical wards.
\end{abstract}

Methods All patients with PD admitted to surgical departments in Aberdeen Royal Infirmary during an 18month period were identified. Medical and nursing notes were reviewed retrospectively, and drug prescription and administration were studied in detail. All documented complications were recorded.

Results 59 surgical admissions ( 51 receiving PD medication, median duration 6 days) were studied. $71 \%$ had missed doses of PD medication, with $34 \%$ missing over $10 \%$ of prescribed doses. Values were similar for levodopa and agonists. Overall, $12 \%$ of all prescribed PD medication was missed (mean 0.7 missed doses per patient per day). No reason for missed doses was recorded in $64 \%$ of cases, while inappropriate reasons included 'out of stock' (12\%) and 'nil by mouth' (8\%). Centrally acting antidopaminergic drugs (mainly antiemetics) were prescribed in $41 \%$ of cases, and administered in $22 \%$. Complications, most commonly neuropsychiatric, were documented in $69 \%$ of nonday-case admissions.

Conclusion Poor prescribing and incomplete drug administration are common in patients with PD on surgical wards. Measures to improve management are identified.

\section{INTRODUCTION}

Parkinsonian syndromes are common, with idiopathic Parkinson's disease (PD) alone probably affecting at least $1 \%$ of the population over 60 years of age. ${ }^{1}$ Many affected people require hospital admission for other medical or surgical problems, and are often treated by staff unfamiliar with managing PD. Suboptimal PD management during such admissions is probably common; in a recent study of 35 emergency admissions in patients with PD (mostly to general medical wards), $74 \%$ of patients had PD medication stopped or adjusted inappropriately, with over half of this group suffering clinically significant sequelae. ${ }^{2}$

Surgical admissions present particular risks. In addition to undergoing surgical procedures and anaesthesia, patients are usually looked after by medical and nursing staff inexperienced in PD management. PD drugs may be abruptly discontinued during 'nil by mouth' periods, and complications such as confusion and nausea may be inappropriately treated with centrally acting antidopaminergic drugs such as haloperidol, metoclopramide and prochlorperazine; these drugs may cause a marked symptomatic deterioration in patients with PD. Despite the potential problems, perioperative management of $\mathrm{PD}$ has not been widely studied. There are anecdotal reports of abrupt cessation of PD drugs in the perioperative period resulting in adverse clinical sequelae, ${ }^{3}$ and a postal survey of patients with PD in Scotland suggested that suboptimal PD management during surgical admissions may be common. ${ }^{4}$ Two studies have also reported increased complication rates in patients with PD after surgery. ${ }^{6}$ To our knowledge, however, no systematic studies of perioperative PD management have been published.

Here, we examined the pharmacological management of patients with PD during surgical admissions, assessing three areas of potential concern: (1) omission of regular PD drugs; (2) timing of levodopa administration; (3) inappropriate prescribing of antidopaminergic drugs. We also measured complication rates and examined the relationship between complications and pharmacological management. In addition, as many hospitals now employ specialist PD nurses (who are able to review patients with PD and provide information and guidance on management), we looked at rates of referral to the PD nurse during these admissions. During the period of our study, there was one PD nurse specialist (LC), who provided a service to the whole of Grampian (population about 500000 ) including inpatients at Aberdeen Royal Infirmary (box 1).

\section{METHODS \\ Patients}

All patients with PD (or parkinsonian syndromes treated with PD medication) admitted under surgical specialties in Aberdeen Royal Infirmary during an 18-month period (November 2002-April 2004) were identified retrospectively through hospital discharge data. Patients were excluded if the diagnosis of parkinsonism was not secure after review of their medical records or if their records had been destroyed after death.

\section{Data collection}

All data were collected retrospectively from hospital records by one author (KJS) and doublechecked by another (CEC). Demographic and clinical data were recorded on all patients, including the normal drug regimen and reason for admission. Medical notes, nursing notes and prescription charts were analysed in detail to identify any missed PD drug doses and any late administration of levodopa (defined as more than $1 \mathrm{~h}$ after the prescribed time). Reasons for missed or late doses were recorded as documented on prescription 
Box 1 Parkinson's disease (PD) nurse service in Aberdeen Royal Infirmary

During the period of this study there was one full-time nurse (LC) whose role was to:

1. Provide information and support to patients newly diagnosed with PD, family members and carers

2. Undertake inpatient and outpatient review of patients with PD under supervision of appropriate consultant (four consultant neurologists and eight geriatricians)

3. Support patients changing their medication

4. Provide direct point of contact for patients, carers and healthcare professionals with hospital PD services

charts. Prescription and administration of antidopaminergic drugs (eg, certain antiemetics, major tranquilisers) was recorded. All documented complications were recorded; these were subdivided into those likely to be directly related to PD (motor or neuropsychiatric complications), and those unlikely to be (such as infections or renal failure). Finally, any documented involvement of the PD specialist nurse during the admission was recorded.

\section{RESULTS}

Sixty-eight patients with admissions under surgical specialities were identified. Fourteen were excluded (notes destroyed in 10 cases, uncertain diagnosis in four). In total, 59 surgical admissions from 54 patients were included in the study. The PD specialist nurse was informed of only one admission (2\%). Patient characteristics and clinical data are summarised in table 1.

\section{Missed and delayed PD medication doses}

Of the 51 admissions in which patients were prescribed PD drugs of any sort, missed doses were documented in $36(71 \%$, $95 \%$ CI $56 \%$ to $83 \%$ ). In 27 admissions (53\%, $95 \%$ CI 39\% to $67 \%$ ), over $5 \%$ of prescribed PD drug doses were missed, and, in 18 (35\%, $95 \%$ CI $22 \%$ to $50 \%$ ), over $10 \%$ of doses were missed. Overall, during these 51 admissions, $12 \%$ of all prescribed PD drugs were missed (this value remained unchanged when data from day cases were excluded). This corresponded to a mean of 0.7 missed doses per patient each day ( 0.6 doses per patient per day when day cases were excluded). Similar results were obtained when only levodopa was considered: missed doses were documented in 33 of 50 admissions (66\%; $95 \%$ CI $51 \%$ to $79 \%$ ), with over $5 \%$ of prescribed levodopa doses missed in 24 admissions (48\%; $95 \%$ CI $34 \%$ to $63 \%$ ), and over $10 \%$ of levodopa administrations missed in 17 admissions (34\%; $95 \%$ CI $21 \%$ to $49 \%)$. Late doses of levodopa were recorded in 13 of 50 admissions ( $26 \%$; $95 \%$ CI $15 \%$ to $40 \%$ ), although documentation of dose timing was generally poor.

Reasons for missed or late doses were documented only sporadically. No reason for being missed or late was recorded for 180 doses (64\%; $95 \%$ CI 49\% to 77\%); for the remainder, reasons documented were 'unable to swallow' in 36 cases (14\%; $95 \%$ CI $7 \%$ to $27 \%$ ), 'out of stock' in 30 (12\%; $95 \%$ CI $5 \%$ to $24 \%$ ), 'nil by mouth' in 21 (8\%; $95 \%$ CI $2 \%$ to $19 \%$ ), 'refused' in eight (4\%; $95 \%$ CI $1 \%$ to $14 \%$ ) and 'in theatre' in eight (4\%; $95 \%$ CI $1 \%$ to $14 \%)$.

\section{Prescribing of inappropriate medication}

Inappropriate antidopaminergic drugs were prescribed in 24 of the 59 admissions ( $41 \%$; $95 \%$ CI $28 \%$ to $54 \%$ ), and administered in 13
Table 1 Characteristics of patients in study

\begin{tabular}{|c|c|}
\hline Patient characteristic & $\begin{array}{l}\text { Study group } \\
(n=54)\end{array}$ \\
\hline Age (years) & $76(49-87)$ \\
\hline Male & $25(47)$ \\
\hline \multicolumn{2}{|l|}{ Diagnosis } \\
\hline - Idiopathic PD & $51(94)$ \\
\hline - Multiple system atrophy & $1(2)$ \\
\hline $\begin{array}{l}\text { Probable drug-related parkinsonism } \\
\text { (but on PD treatment) }\end{array}$ & $2(4)$ \\
\hline \multicolumn{2}{|l|}{ Stage of parkinsonism ${ }^{7}$} \\
\hline $\begin{array}{l}\text { - Stage } 1 \text { (diagnostic) } \\
\text { Stage } 2 \text { (maintenance) } \\
\text { - Stage } 3 \text { (complex) } \\
\text { Stage } 4 \text { (palliative) }\end{array}$ & $\begin{aligned} 8 & (15) \\
19 & (35) \\
25 & (46) \\
1 & (2)\end{aligned}$ \\
\hline Time since diagnosis & $5(1-20)$ \\
\hline \multicolumn{2}{|l|}{ Treatment } \\
\hline $\begin{array}{l}\text { No treatment } \\
\text { Levodopa preparations } \\
\text { Dopamine agonists } \\
\text { Other PD medication }\end{array}$ & $\begin{array}{r}8(14) \\
50(85) \\
18(31) \\
14(24)\end{array}$ \\
\hline \multicolumn{2}{|l|}{ Type of admission } \\
\hline $\begin{array}{l}\text { Elective } \\
\text { Emergency }\end{array}$ & $\begin{array}{l}26(44) \\
33(56)\end{array}$ \\
\hline Day cases & $23(39)$ \\
\hline \multicolumn{2}{|l|}{ Duration of admission } \\
\hline $\begin{array}{l}\text { All patients } \\
\text { All patients excluding day cases } \\
\text { - Emergency admissions } \\
\text { - Elective admissions }\end{array}$ & $\begin{array}{r}6(1-90) \\
10.5(2-90) \\
10(1-90) \\
1.5(1-26)\end{array}$ \\
\hline \multicolumn{2}{|l|}{ Surgical specialty of admission } \\
\hline - Orthopaedics & $24(41)$ \\
\hline - General surgery & $17(29)$ \\
\hline - Ophthalmology & $6(10)$ \\
\hline - Cardiothoracic & $3(5)$ \\
\hline Other & $9(15)$ \\
\hline
\end{tabular}

(22\%; $95 \%$ CI $12 \%$ to $35 \%$ ), although usually only one or two doses were given; in some cases more than one such drug was prescribed. Inappropriate drugs included prochlorperazine in 20 patients $(34 \%)$, cyclizine in seven patients $(12 \%)$, metoclopramide in two patients (3\%) and haloperidol in two patients (3\%).

\section{Complications}

Complications were common, occurring in 28 cases overall ( $47 \%$; $95 \%$ CI $28 \%$ to $54 \%$ ); a breakdown of complication types is given in table 2 . When day cases were excluded $(n=23)$, the proportion of admissions associated with complications was $69 \%$ ( $95 \%$ CI $56 \%$ to $81 \%$ ). Neuropsychiatric complications (confusion, hallucinations and agitation) were seen in $52 \%$ of this nonday-case case group (95\% CI 36\% to 70\%). Motor deterioration in parkinsonism was noted in 7\% (95\% CI $2 \%$ to $17 \%)$, with falls in a further $10 \%$ (95\% CI $4 \%$ to $21 \%$ ). Other general medical complications (including infection, cardiac arrhythmias and renal impairment) were recorded in $26 \%$ (95\% CI $15 \%$ to $38 \%$ ). One patient died from sepsis and multiorgan failure. The frequency of overall complications appeared to increase with increasing proportion of missed PD doses (figure 1).

\section{DISCUSSION \\ Principal findings}

To our knowledge, this is the first systematic study of inpatient PD medication management during surgical admissions. Management was often suboptimal; PD medication was missed at a mean rate of 0.7 doses per day, late administration of drugs was common, and centrally acting antidopaminergic drugs were 
Table 2 Summary of complications observed in patients with Parkinson's disease (PD) during admissions, and frequency expressed in terms of (1) number of admissions and (2) percentage of all admissions (including day cases) $(n=59)$

\begin{tabular}{lc}
\hline Complication & $\begin{array}{l}\text { No of } \\
\text { admissions } \\
\text { with } \\
\text { complication } \\
\text { (\% of all } \\
\text { admissions) }\end{array}$ \\
\hline Neuropsychiatric (confusion/agitation/aggression/hallucinations) & $24(41)$ \\
Worsening motor PD symptoms (bradykinesia/rigidity) & $3(5)$ \\
Falls & $5(8)$ \\
Other (atrial fibrillation, heart failure, renal failure, & $11(19)$ \\
infection, hypotension) & \\
\hline
\end{tabular}

prescribed (although not always given) in almost half of the admissions. The PD specialist nurse was underutilised, being informed in only $2 \%$ of admissions. Complications, particularly neuropsychiatric symptoms, were common, occurring in $69 \%$ of non-day-case admissions. Complications were most common in patients in whom the greatest proportion of prescribed $\mathrm{PD}$ medication was missed.

The findings appear to reflect a paucity of knowledge and experience in managing patients with PD in surgical departments. For example, the widespread prescribing of centrally acting antidopaminergic drugs suggests a lack of understanding on the part of medical staff regarding the potential impact of these drugs in PD. Likewise, inappropriate reasons for missed PD drug doses such as 'out of stock', 'nil by mouth' and 'unable to swallow' were common, reflecting unfamiliarity with $\mathrm{PD}$ among nursing staff. It is good practice for essential oral drugs to be continued during a 'nil by mouth' period, and PD medications should be given at this time to prevent symptomatic deterioration, particularly in patients with more advanced disease. Nasogastric tubes can be used early in patients who have difficulties with swallowing or are too drowsy to take their oral medication. Furthermore, although surgical wards may often not routinely stock PD drugs, they will be available elsewhere in the hospital.

\section{Study design}

Although this study identified widespread deficiencies in $\mathrm{PD}$ management, the retrospective study design limited the degree of information available. However, the data probably underestimate

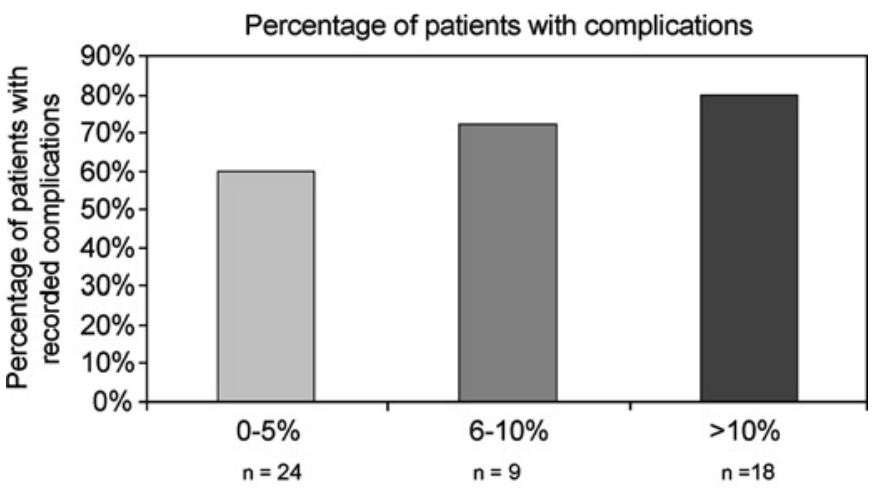

Groups of patients subdivided by percentage of missed PD medication during inpatient stay ( $n=$ number of patients with each level of missed medication)

Figure 1 Relationship between the proportion of missed levodopa doses during admission ( $x$ axis) and the rate of documented complications (y axis). Higher complication rates are evident in those patients who missed the greatest proportion of their Parkinson's disease (PD) medication. the scale of the problem. In particular, the rates of late drug administration reported here were, almost certainly, a substantial underestimate. In many patients with $\mathrm{PD}$, particularly those with stage 3 (complex) disease, the presence of end-of-dose phenomena make the timing of PD drug administration critical. Many drugs doses may have been given late but not recorded so, particularly if the drug round itself started on time.

The study design also makes it impossible to establish a causal relationship between complications and missed PD drug doses. The overall rate of documented complications (69\% of non-daycase admissions) was high and increased with a greater proportion of missed medication. However, the numbers were too small to make meaningful statistical comparisons, and it is not possible to comment on causality; missed drug doses may have been either a consequence or cause of complications. A prospective study would be required to investigate this relationship further.

\section{Practical recommendations}

Despite limitations resulting from the retrospective study design, these data have clearly highlighted problems in the perioperative management of patients with PD. From a practical perspective we have therefore made a number of recommendations, many of which are now in the process of implementation in our hospital.

Possibly the most effective and feasible strategy is to increase the input of specialist PD services in the surgical setting; specialist PD nurses, now present in many hospitals, may be the ideal point of contact. It is possible to generate computerised

\section{Main messages}

1. Inappropriate withholding of Parkinson's disease (PD) medications is common in patients with $P D$ during surgical admissions.

2. Prescription of contraindicated antidopaminergic drugs (particularly antiemetics) is also common, although actual administration is less common.

3. Mechanisms to avoid these problems and optimise management of patients with PD during surgical admissions should be considered including:

a. Routinely contacting the PD nurse (either automatically through patient administration systems or giving patients a 'PD card' to give to staff on admission, to enable early access to specialist PD services).

b. Focused education in key areas (eg, orthopaedic wards, pharmacy).

c. Reducing the routine use of centrally acting antidopaminergic drugs for nausea.

d. Promoting patient self-medication.

\section{Current research questions}

1. Will the institution of the measures to improve medication management reduce the rate of missed medication and inappropriate prescribing during hospital admissions?

2. Does improving inpatient Parkinson's disease (PD) medication management have a significant impact on clinical outcomes including complication rates and length of stay? 
alert systems (triggered via patient administration systems) to inform the PD nurse of a surgical admission, thus enabling active involvement early in the course of the admission. This does, however, require a regularly updated database of local patients with PD as well as sufficient numbers of PD nurses. As an additional measure, patients can be given a 'warning card' to present to medical or nursing staff at the time of any hospital admission, stating the diagnosis of PD and advising the admitting team to contact the local PD nurse. This would enable surgical staff to receive timely advice regarding the importance of regular prescribed medication (even during 'nil by mouth' periods, or when the patient is too drowsy to swallow) and avoid inappropriate drug prescription.

Staff education may have some benefits, but as the proportion of patients with PD admitted to most wards is very small and the turnover of staff high, this may have minimal impact. Focusing education in particular areas (eg, orthopaedic wards, hospital pharmacists) may be the most effective strategy. In addition, more general educational measures, such as underlining the importance of continuing all essential medication during 'nil by mouth' periods and encouraging the use of domperidone over centrally acting antidopaminergic drugs as a first-line antiemetic, may be helpful.

Finally, more widespread use of self-administration of drugs can be useful in some patients. Although this will not always be possible, particularly when confusion or somnolence is a significant problem, in some it may enable accurate timing of doses.

In practice, a combination of these practices may minimise the risk of iatrogenic worsening of PD symptoms during surgical admissions and potentially reduce complication rates and duration of hospital stay. Prospective studies are, however, required if a clear evidence base for optimisation of perioperative management in $\mathrm{PD}$ is to be developed.

Competing interests None declared.

Provenance and peer review Not commissioned; externally peer reviewed.

\section{REFERENCES}

1. Campenhausen S, Bornschein B, Wick R. Prevalence and incidence of Parkinson's disease in Europe. Eur Neuropsychopharmacol 2005;15:473-90.

2. Magdalinou K, Martin A, Kessel B. Prescribing medications in Parkinson's disease (PD) patients during acute admissions to a district general hospital. Parkinsonism Relat Disord 2007:13:539-40.

3. Kennedy JM. Polypharmacy in a general surgical unit and consequences of drug withdrawal. Br J Clin Pharmacol 2000;49:353-62.

4. Barber M, Stewart D, Grosset D, et al. Patient and carer perception of the management of Parkinson's disease after surgery. Age Ageing 2001;30:171-2.

5. Golden W, Lavender R, Metzer W. Acute postoperative confusion and hallucinations in Parkinson disease. Ann Intern Med 1989;111:218-22.

6. Pepper P, Goldstein M. Postoperative complications in Parkinson's disease. J Am Geriatr Soc 1999:47:967-72.

7. MacMahon D, Thomas S. Practical approach to quality of life in Parkinson's disease: the nurse's role. J Neurol 1998;245(Suppl 1):S19-22. 